\title{
Concepto de Developmental Origins of Health and Disease: El ambiente en los primeros mil días de vida y su asociación con las enfermedades no transmisibles \\ Developmental Origins of Health and Disease Concept: The environment in the first 1000 days of life and its association with noncommunicable diseases
}

\author{
Subcomisión DOHaD - SAP "Origen de la Salud y Enfermedad en el Curso de la \\ Vida" - Sociedad Argentina de Pediatría*
}

\section{RESUMEN}

En las últimas décadas, las enfermedades crónicas no transmisibles (ENT) se han convertido en la principal causa de mortalidad global y han aumentado en América Latina. La contribución de recursos de la ciencia del desarrollo, la epigenética, las neurociencias, las ciencias ambientales, la epidemiología y la investigación ha generado evidencia del origen de las ENT desde la programación fetal. Los resultados de salud y enfermedad devienen de una trayectoria dinámica en la que se agregan factores protectores para una vida saludable o factores de riesgo para enfermedades del individuo y las comunidades.

El concepto de Developmental Origins of Health and Disease redimensiona el papel del equipo de salud materno-infantil y debe guiar las políticas públicas para priorizar los primeros mil días de vida para un desarrollo saludable y la prevención de ENT. Se presenta una actualización sobre las principales condiciones ambientales adversas que pueden alterar la programación del desarrollo y predisponer a ENT en el curso de la vida.

Palabras clave: exposición a riesgos ambientales, enfermedades no transmisibles, desarrollo infantil, epigenómica, medioambiente y salud pública.

\section{ABSTRACT}

In recent decades, chronic non-communicable diseases (NCDs) have become the leading cause of global mortality and increased in Latin America. The contribution of the resources from development science, epigenetics, neurosciences, environmental sciences, epidemiology and research has generated evidence of the origin

Correspondencia:

Dra. Stella Maris Gil:

stellamgil@gmail.com

\section{Financiamiento:}

Ninguno.

Conflicto de intereses:

Ninguno que declarar.

Recibido: 7-2-2020

Aceptado: 18-2-2020 and disease outcomes result from a dynamic trajectory where protective factors are added for a healthy life or risk factors for diseases of the individual and the communities. Developmental Origins of Health and Disease concept resizes the role of the maternal and child health team and should guide public policies by prioritizing the first 1000 days of life for healthy development and NCDs prevention. We present an update on principal adverse environmental conditions that may alter development programming and predispose NCDs in life course.

Key words: environmental exposure, noncommunicable diseases, child development, epigenomics, environment and public health.

http: / / dx.doi.org/10.5546/ aap.2020.S118

Cómo citar: Subcomisión DOHaD - SAP “Origen de la Salud y Enfermedad en el Curso de la Vida" Sociedad Argentina de Pediatría. Concepto de Developmental Origins of Health and Disease: El ambiente en los primeros mil días de vida y su asociación con las enfermedades no transmisibles. Arch Argent Pediatr 2020;118(4):S118-S129.

\section{INTRODUCCIÓN}

El concepto de Developmental Origins of Health and Disease (DOHaD), acrónimo inglés cuya traducción al español significa "origen de la salud y enfermedad en el desarrollo", fue descrito originalmente en la década
* Dr. Jorge Cabana, Dra. Débora Sabatelli, Dra. Miriam Tonietti, Dra. Angélica Flores, Dra. Roxana Conti, Dra. Diana Pasqualini, Dra. Laura Gaete, Dra. Stella Maris Gil.

Colaboradores: Dra. Blanca Ozuna, Dr. Carlos Grandi, Dra. Patricia Kaminker, Dra. Patricia Jáuregui Leyes, Dra. Marcela Regnando, Dra. Gisela Martinchuk. 
de los ochenta por el Dr. David Barker al explicar la relación de un bajo peso de nacimiento como consecuencia de un ambiente adverso nutricional y la enfermedad cardiovascular en la vida adulta. Desde entonces, las investigaciones se han ido extendiendo hacia otras disciplinas y las evidencias sobre la asociación entre un ambiente adverso desde los inicios de la vida y el desarrollo posterior de enfermedades no transmisibles (ENT) han permitido la comprensión del origen de algunas enfermedades metabólicas, como diabetes y obesidad, ciertos tipos de cáncer y algunos trastornos en la salud reproductiva, en la salud mental y en el neurodesarrollo.

Las ENT son responsables de más del $60 \%$ de las muertes en el mundo y ocasionan alrededor del $80 \%$ de las defunciones en la región de las Américas. Su incidencia se ha incrementado de modo abrupto en las últimas dos décadas $\mathrm{y}$, aunque, en el inicio, estas enfermedades ocurrieron, sobre todo, en países industrializados, son particularmente prevalentes en los países poco desarrollados que atraviesan mejorías socioeconómicas. ${ }^{1,2}$

Si bien se reconoce que pueden prevenirse reduciendo los 4 principales factores de riesgo, el consumo de tabaco, el consumo nocivo de alcohol, una alimentación poco saludable y la inactividad física, la incorporación del concepto $\mathrm{DOHaD}$ se enfoca en la prevención de un ambiente adverso desde el origen de la vida. $D O H a D$ se enmarca en un modelo integrador de "ecobiodesarrollo", ${ }^{3}$ que explica cómo las influencias ambientales tempranas, reconocidas como la "ecología del individuo" (el entorno ambiental, familiar, hábitos, condiciones socioeconómicas y el estrés tóxico), pueden afectar el programa biológico (herencia genética) y dar como resultado fenotipos que manifiestan alteraciones en las capacidades de aprendizaje, en los comportamientos adaptativos, la salud física y mental en el curso de la vida.

Las experiencias que modelan el desarrollo comienzan en la etapa preconcepcional e impactan especialmente en los "mil días de vida" (período gestacional y los dos primeros años de vida posnatal). Esta es la etapa más sensible, cuando se producen modificaciones epigenéticas que provocarán efectos en el curso de la vida del individuo y pueden transmitirse a las siguientes generaciones.

Es sabido que existe una estrecha interacción entre nuestros genes y el entorno. Los resultados de estudios experimentales y en humanos muestran que las características del entorno en la vida temprana juegan un papel importante en la configuración del fenotipo del individuo como una respuesta adaptativa a los estímulos que recibió durante el desarrollo. Esta adaptación se realiza a través de la regulación epigenética, mediante procesos químicos que no alteran la secuencia del ácido desoxirribonucleico (ADN) y permiten la expresión o silenciamiento de los genes, lo que origina diversos fenotipos. Si bien son procesos dinámicos y reversibles, tienden a estabilizarse con la edad.

La etapa del desarrollo constituye un período de gran plasticidad tisular y de actividad epigenética muy sensible a las perturbaciones ambientales, en especial, a la malnutrición, el estrés, las drogas y los contaminantes ambientales. Haugen y cols., plantean, luego de 20 años de investigación y seguimiento, una visión amplia de los orígenes del desarrollo de la salud y la enfermedad, y reafirman los últimos datos que muestran la transmisión transgeneracional de la susceptibilidad a la enfermedad. ${ }^{4}$

Aunque los esfuerzos de la prevención para reducir las ENT se han centrado, principalmente, en los adultos y en factores de riesgo (sobre todo, malnutrición, inactividad física, consumo de tabaco y alcohol), la evidencia en apoyo del paradigma de $\mathrm{DOHaD}$ sugiere que es necesario focalizarse en la optimización de la nutrición, en la reducción del estrés y de las exposiciones a tóxicos ambientales en etapas tempranas de la vida. ${ }^{2}$ La adopción del paradigma de $\mathrm{DOHaD}$ puede promover un enfoque más realista, preciso e integrador del proceso de salud/enfermedad originado en la programación del desarrollo y permite orientar mejor las intervenciones clínicas y las políticas de salud. Así, el período del desarrollo es una etapa de oportunidades en la que se puede promover una mejor salud para toda la vida teniendo presente que "un mal comienzo dura toda una vida, pero un buen comienzo también".

En esta actualización, se presentan evidencias científicas seleccionadas que brindarán al equipo de salud información necesaria para entender el concepto de $D O H a D$ y los mecanismos que llevan al desarrollo de las ENT durante el curso de la vida. Esto facilitará la toma de decisiones orientadas a su prevención en todos los niveles, desde la atención primaria hasta la orientación de las políticas públicas en salud. Este documento está alineado con el Plan de acción global para la prevención y control de enfermedades no transmisibles 
de la Organización Mundial de la Salud (OMS) y con los "Objetivos de desarrollo sustentable" adoptados por las Naciones Unidas para la Agenda 2030.

\section{GLOSARIO}

Epigenética: El término significa literalmente "por encima del ADN". Es la rama de la biología que estudia las interacciones entre los genes y el ambiente, que dan origen al fenotipo. Es un sistema de regulación que modera la expresión de los genes a través de mecanismos moleculares que controlan la actividad genética, sin afectar la composición del ADN. Estos mecanismos son heredables por mitosis y meiosis, y dinámicos, o sea, pueden ser reversibles.

Mecanismos epigenéticos: Los mecanismos epigenéticos se basan en modificaciones bioquímicas del ADN de manera estable y heredable sin cambiar la secuencia de ADN subyacente. Las modificaciones epigenéticas comúnmente estudiadas en la cromatina incluyen metilación del ADN, modificaciones de histonas y micro ácido ribonucleico (micro-ARN) o ARN no codificantes.

Plasticidad fetal: Es el proceso a través del cual un estímulo o insulto en un período crítico o sensible puede modificar el desarrollo fetal con posibles efectos en el largo plazo.

\section{Developmental Origins of Health and Disease y nutrición temprana}

Tradicionalmente, las ENT eran consideradas el resultado de la asociación entre un perfil genético determinado y factores del estilo de vida en la adultez. En la actualidad, se reconoce la importancia del ambiente temprano, pre- y posnatal, en la determinación de la susceptibilidad para el desarrollo de tales enfermedades a lo largo de la vida.

Los fenotipos alterados se inducen a través de mecanismos genéticos, fisiológicos (especialmente, endócrinos) y epigenéticos (interacción entre genes y ambiente). Si bien las variaciones genómicas de riesgo son responsables de una pequeña proporción en la prevalencia de las ENT, el rápido incremento en la incidencia no permite explicar que este aumento sea exclusivamente por cambios del genoma. ${ }^{5}$

La teoría del curso vital que permite comprender el desarrollo de las enfermedades en la adultez señala que el riesgo de enfermarse aumenta a lo largo de la vida como consecuencia de una disminución de la plasticidad fenotípica y los efectos acumulativos resultantes de respuestas inadecuadas frente a desafíos continuados del medioambiente. Las modificaciones del epigenoma (alteraciones en la metilación del ADN, modificaciones covalentes de las histonas y los ARN no codificantes) regulan la adaptación al ambiente del organismo en desarrollo, quien ajusta sus sistemas metabólicos y homeostáticos para adaptarse al medio extrauterino en forma anticipada. Estos procesos epigenéticos tienen un rol principal en la regulación de la expresión génica específica en los distintos tejidos. De esta manera, las alteraciones en estos procesos son capaces de inducir cambios duraderos en la expresión de genes y en el metabolismo que persisten a lo largo de la vida. ${ }^{6,7}$

Diversas investigaciones avalan la asociación entre la calidad del medioambiente en la vida temprana y el riesgo futuro de enfermedad en la vida adulta. Las primeras descripciones fueron realizadas por Forsdahl en 1977, quien comunicó la asociación directa encontrada entre las tasas de mortalidad infantil y el riesgo cardiovascular en la adultez. ${ }^{8}$

Los estudios de Ravelli y cols., demostraron los efectos de la nutrición temprana sobre la susceptibilidad a enfermarse posteriormente en la vida. Durante la hambruna holandesa que tuvo lugar en el invierno de 1944, los individuos cuyas madres estuvieron expuestas a la restricción alimentaria (dietas de 600-800 calorías / día) periconcepcionalmente y en el primer trimestre de la gestación, aunque no tuvieron reducción en el peso al nacer, presentaron mayor riesgo de obesidad y enfermedad cardiovascular en la adultez. Aquellos individuos cuyas madres estuvieron expuestas a la restricción en la segunda mitad de la gestación fueron más pequeños al nacer y con un aumento de la incidencia de insulinorresistencia e hipertensión arterial posteriormente. ${ }^{9}$ No solo estos cambios en el fenotipo fueron resultado de la subnutrición, sino que también se han demostrado efectos epigenéticos y riesgo para el desarrollo de enfermedades metabólicas con la sobrenutrición temprana.

Luego, el grupo del profesor Barker, en el Reino Unido, encontró una relación inversa entre el peso al nacer y la mortalidad cardiovascular en los adultos. A partir de allí, numerosos estudios epidemiológicos retrospectivos reprodujeron los resultados de la asociación entre el peso al nacer y el riesgo cardiovascular y también con el riesgo de desarrollo de hipertensión, obesidad y diabetes 
mellitus tipo 2 (DM2). Más tarde, se reconoció que el peso al nacer era un indicador demasiado crudo para representar el ambiente intrauterino, que podía resultar afectado por una gran variedad de factores maternos, placentarios o ambientales (estado nutricional materno, alimentación antes y durante el embarazo, tabaco, tóxicos, estrés). ${ }^{10}$

El Estudio de niños de Pune, India, fue la primera investigación colaborativa que confirmó que el bajo peso al nacer estaba asociado con el desarrollo de insulinorresistencia tan temprano como a los 4 años de vida. En esta población, tanto a los 4 como a los 8 años, la insulinorresistencia estaba estrechamente relacionada con un peso más bajo al nacer. ${ }^{11,12}$

Luego, se diseñó el Estudio de la nutrición materna en Pune (1993), con el objetivo de investigar la influencia de la nutrición materna durante el embarazo sobre el crecimiento fetal y el riesgo posterior. ${ }^{13}$ Se evaluaron más de 800 embarazos; los niños fueron visitados cada 6 meses con determinaciones antropométricas y, cada 6 años, padres y niños fueron investigados en su composición corporal, factores de riesgo cardiometabólico y desarrollo cognitivo. En promedio, las madres del estudio tenían 21 años, pesaban $42 \mathrm{~kg}$ (índice de masa corporal -IMC-: $18,1 \mathrm{~kg} / \mathrm{m}^{2}$ ) y recibían dietas con $1700 \mathrm{kcal}$ y $45 \mathrm{~g}$ de proteínas por día. Los recién nacidos (RN), en promedio, pesaron $2700 \mathrm{~g}$ y el $28 \%$ fueron menores de $2500 \mathrm{~g}$.

Los hijos de madres con mayor peso eran más grandes, y los niños de las madres más altas fueron de mayor longitud. Se encontró una correlación entre el tamaño del padre y la longitud del niño (crecimiento esquelético), mientras que la adiposidad estuvo, fundamentalmente, determinada por factores maternos. Las madres con menor talla y mayor adiposidad tuvieron niños más adiposos (efecto intergeneracional). La ganancia de peso gestacional y el peso placentario a las 18 semanas de gestación se correlacionó con las medidas antropométricas del RN, lo que reveló la importancia de la nutrición maternofetal temprana y el rol de la placenta. Estos estudios muestran cómo estos efectos pueden programarse en la vida fetal.

Es sabido que la vitamina B12 es un nutriente muy importante para el desarrollo del sistema nervioso central (SNC) y que colabora como cofactor en las reacciones de metilación del organismo. En el estudio de Pune, se evidenció que la función cognitiva (memoria y atención) de los niños a los 9 años fue peor en aquellos en los que sus madres habían tenido baja concentración de vitamina B12 a las 28 semanas de gestación, comparados con los hijos de madres con el mayor valor de vitamina B12. ${ }^{14}$

Hay suficiente evidencia de que la obesidad materna está asociada al aumento de la obesidad, DM2 y enfermedad cardiovascular en los hijos. Más allá de compartir genes obesogénicos entre madre e hijos, los resultados sobre la importancia del ambiente materno en estas asociaciones son contundentes. Primero, se reconoce una mayor asociación entre el IMC de la madre y el IMC del hijo que con el IMC del padre. Estudios de hermanos nacidos antes y después de la cirugía bariátrica en madres con obesidad demuestran la reducción del peso al nacer y el riesgo de obesidad en los nacidos poscirugía. ${ }^{15}$

Los efectos de las alteraciones del crecimiento fetal sobre la salud metabólica posterior se ven exacerbados si ocurre un crecimiento acelerado posnatal (catch up) u obesidad. En la cohorte de Hertfordshire, los hombres que tuvieron mayores alteraciones en la tolerancia a la glucosa fueron los de menor peso al nacer y que, a los 64 años, estaban obesos. ${ }^{16}$ Posteriormente, dos estudios demostraron relaciones entre el bajo peso al nacer, la rápida ganancia de peso en la infancia y el riesgo de alteraciones en la tolerancia a la glucosa y diabetes. ${ }^{17,18}$

Hay estudios que demuestran la importancia de la calidad nutricional posnatal temprana y el riesgo de obesidad: los niños amamantados tienen menor riesgo de desarrollar obesidad que los alimentados con fórmula. Menor concentración de proteína en la fórmula se asocia con menor ganancia de peso en los niños a los 2 años. ${ }^{19}$ Estudios experimentales aleatorizados han demostrado la reducción del riesgo de obesidad y de factores de riesgo cardiovascular en los niños con ingestas modificadas de nutrientes durante el período neonatal. ${ }^{20}$

Otra población de riesgo en la actualidad son los individuos que han sido RN prematuros. Los estudios epidemiológicos iniciales no distinguían entre retardo del crecimiento intrauterino (RCIU) y prematuridad como causantes del bajo peso de nacimiento. En la actualidad, hay evidencia de que la prematuridad en sí misma es un factor de riesgo para el desarrollo de ENT en etapas posteriores de la vida. El crecimiento compensatorio en peso es, generalmente, mayor que para la talla. La alteración de la distribución de la masa grasa descrita en la infancia de los niños nacidos pretérmino podría persistir en la adultez. ${ }^{21}$ 
Las experiencias nutricionales tempranas relacionadas con la salud materna, el ambiente intrauterino y la alimentación en los primeros dos años de vida proveen un marco adecuado para el conocimiento de cómo las diferencias en el ambiente durante el desarrollo temprano pueden llevar a cambios permanentes en el metabolismo. Estos eventos programan riesgos para trastornos crónicos, tales como obesidad, síndrome metabólico, enfermedad cardiovascular, enfermedades neurodegenerativas y cognitivas.

\section{Developmental Origins of Health and Disease y tóxicos ambientales}

La teoría de Barker abrió una gran puerta para la investigación de otras disciplinas, como las ciencias ambientales. La exposición del individuo a productos químicos tóxicos y otros factores de estrés ambiental es omnipresente y se ha demostrado que, antes de la concepción, durante la gestación y en la vida posnatal, puede tener un impacto grave en la salud que aparece luego de un período de latencia de varios años.

Se ha documentado que la exposición prenatal a algunas sustancias químicas aumenta el riesgo de cáncer en la infancia; otras producen perturbación hormonal; la exposición a los plaguicidas se ha relacionado con alteraciones en la calidad del semen en el hombre adulto, esterilidad y cáncer de próstata, y puede interferir con todas las etapas de desarrollo de la función reproductiva en las mujeres adultas, la pubertad, la menstruación y la ovulación, la fertilidad, la fecundidad y la menopausia. Muchos factores ambientales perjudiciales para la salud reproductiva afectan de modo particular a las poblaciones más vulnerables y marginadas.

La evidencia sobre la exposición a agentes ambientales tóxicos y los resultados adversos en la salud reproductiva y el desarrollo es suficientemente extensa y sólida. Así, el Colegio Norteamericano de Obstetricia y Ginecología y la Sociedad Americana de Medicina Reproductiva plantean que la reducción de la exposición a agentes ambientales tóxicos es un área crítica de intervención para obstetras, ginecólogos y otros profesionales de la salud reproductiva, quienes debieran desarrollar medidas oportunas para identificar y reducir la exposición de sus pacientes a dichos tóxicos, especialmente, en los primeros mil días de vida. ${ }^{22}$

Otro de los aspectos más estudiados en los últimos 10 años es el rol de la microbiota intestinal en el metabolismo, los químicos ambientales y su relación con el proceso de salud y enfermedad del huésped. Es sabido que la diversidad microbiana se adquiere muy temprano en la vida dentro de las primeras horas después del nacimiento y que el perfil microbiano depende de factores que incluyen el genotipo, el tipo de parto (cesárea o vaginal), la terapia antibiótica temprana, la composición de la dieta, el estilo de vida, las interacciones sociales y la exposición ambiental a varios xenobióticos. Según investigaciones recientes, la disbiosis intestinal provocada por estos factores se asocia a fenotipos asociados a distintas enfermedades del sistema gastrointestinal, neurológico, trastornos metabólicos y de la inmunidad. ${ }^{23}$

La llegada de tecnologías mejoradas de secuenciación de ADN permitió una evaluación más exhaustiva de la composición de la microbiota, funciones y comportamiento frente a los químicos ambientales. Se la puede considerar un órgano con un nivel de actividad productora y depuradora comparable con la del hígado. Además de proteger contra patógenos e intervenir en la regulación de otras funciones fisiológicas del organismo, los genes microbianos modulan la biotransformación de xenobióticos y afectan su biodisponibilidad o toxicidad. Pueden transformar un químico a través de las enzimas microbianas o de sus metabolitos y hacerlo más o menos tóxico. A su vez, los químicos pueden modificar el perfil microbiano y producir una disbiosis que rompe la homeostasis necesaria para mantener el estado de salud.

Algunos químicos ambientales son metabolizados por la microbiota gastrointestinal y generan otros efectos tóxicos o metabolitos con distinto potencial. Estudios experimentales $\mathrm{y}$ en animales demostraron transformaciones de hidrocarburos aromáticos policíclicos (HAP) en moléculas estrogénicas después de la exposición a una microbiota humana típica, lo que sugiere que los microorganismos presentes en el colon humano pueden bioactivar los HAP y convertirlos en moléculas con un potencial que originariamente no poseen. ${ }^{24}$

Otros estudios en modelos animales demostraron que la exposición a nanopartículas, contaminantes del aire (PM10) y metales pesados, como el plomo, el hierro y el arsénico, altera la diversidad y abundancia microbiana, aumenta la población de esporas bacterianas, modifica el metaboloma de la microbiota con mayor secreción de citocinas proinflamatorias, elimina la biopelícula bacteriana a lo largo del 
revestimiento de la mucosa intestinal y altera las vías metabólicas (que involucran la vitamina $\mathrm{E}$, los ácidos biliares, el metabolismo del nitrógeno, el metabolismo energético, el estrés oxidativo y los mecanismos de defensa/desintoxicación). ${ }^{25-27}$

De la misma manera, se han visto modificaciones en la composición microbiana con la exposición a disruptores endócrinos, como el bisfenol A (BPA), estradiol (E2) o etinilestradiol, estrógeno en las píldoras anticonceptivas, lo que aumenta las bacterias asociadas con diversas enfermedades, como la enfermedad intestinal inflamatoria (EII), trastornos metabólicos y cáncer colorrectal. ${ }^{28,29}$

La disbiosis intestinal por tóxicos ambientales también puede provocar trastornos neuroconductuales. El aumento de la permeabilidad intestinal producido por la interacción del tóxico con las bacterias permite que las bacterias y sus metabolitos penetren a través del revestimiento intestinal e invadan los vasos sanguíneos subyacentes. Pueden producir efectos en el SNC por vía sistémica y estimular la liberación de citoquinas inflamatorias o vagal a través de la liberación de neurotransmisores microbianos. ${ }^{30}$

Algunos ejemplos de metabolitos bacterianos que tienen vínculos estrechos con la disfunción cognitiva son los ácidos grasos de cadena corta (short-chain fatty acids; SCFA, por sus siglas en inglés), como el propionato y butirato, presentes en grandes cantidades en la materia fecal de los pacientes con trastornos del espectro autista (TEA). ${ }^{31,32}$ El trabajo futuro en toxicología requerirá una mayor comprensión de cómo interactúan los tóxicos ambientales con el microbioma humano, cómo afectará el perfil y metaboloma microbiano, la epigenética y, por ende, la salud de las futuras generaciones.

\section{Developmental Origins of Health and Disease y lactancia materna}

La leche materna es la nutrición por excelencia para los RN y los niños pequeños, resultado de millones de años de evolución. Además de su valor nutricional, proporciona una variada microbiota e innumerables componentes biológicamente activos que, se cree, guían el desarrollo del sistema inmune del niño. Las bacterias del intestino de la madre pueden ser transferidas a la leche materna y pasar al bebé. Esta interacción es clave para establecer un microbioma intestinal saludable cuyas bacterias protegen contra enfermedades respiratorias y diarreicas, pero que también están sujetas al estrés, al uso de antibióticos o los tóxicos ambientales. ${ }^{33}$

Los oligosacáridos de la leche humana (LH), cuya síntesis está parcialmente determinada por el genotipo materno, juegan un papel en la prevención de la adhesión bacteriana patógena a través de múltiples mecanismos, mientras que también proporcionan nutrición para el microbioma.

Uno de los componentes de la leche materna más recientemente identificados, que tiene implicancia en la respuesta inmune intestinal y la conformación de la microbiota intestinal, son las vesículas extracelulares. Estas contienen una importante carga proteica, capaz de influir en la respuesta inmune local frente a la exposición bacteriana. Las vesículas extracelulares contienen ARN y proteínas de membrana y citosólicas, que están involucradas en la señalización intercelular y desempeñan un papel adicional en el desarrollo del lactante.

La LH tiene abundancia de especies de Bifidobacterium y Lactobacillus. Las bifidobacterias se reducen ante una corta duración de la lactancia materna o el uso temprano de antibióticos y han demostrado protección contra la obesidad y las enfermedades metabólicas en roedores. En los humanos, se observó que la obesidad y los marcadores metabólicos asociados se correlacionaban negativamente con la abundancia de Bifidobacterium.

En los niños, el aumento de Firmicutes y la disminución de bifidobacterias después del destete se han asociado con un aumento de peso y adiposidad. Las bacterias pertenecientes al grupo Clostridium, cuya abundancia relativa se incrementa en los niños con destete precoz o uso temprano de antibióticos, pueden promover la ganancia de peso. ${ }^{34}$

Una microbiota dominada por oligosacáridos y bifidobacterias de la LH se asocia con un desarrollo óptimo de la barrera intestinal, reducción de lipopolisacáridos circulantes, menor inflamación y aumento del metabolismo de los ácidos biliares. La menor duración de la lactancia materna y el uso de antibióticos antes del destete están asociados con una microbiota cuya composición se relaciona con el incremento de peso excesivo. ${ }^{35}$

La composición de macronutrientes de la LH depende de una variedad de factores, que incluyen el ambiente materno, la etapa de lactancia, la dieta materna, la frecuencia de 
lactancia e IMC materno. Los macronutrientes de la LH proporcionan apoyo para el desarrollo de una microbiota protectora en el intestino infantil. Específicamente, las proteínas glicosiladas que pasan a través de la LH actúan como un mecanismo de defensa contra potenciales patógenos dentro del intestino durante la vida pediátrica.

Las hormonas presentes en la LH también contribuyen a la función de barrera intestinal neonatal, ya que reducen la inflamación intestinal y favorecen el normal desarrollo de la mucosa intestinal. El contenido y la calidad de los macronutrientes y hormonas reguladoras del apetito presentes en la LH pueden afectar la biología infantil y el comportamiento alimentario. Las hormonas reguladoras del apetito, como la adiponectina, leptina, insulina, grelina y resistina, están presentes en la LH y han sido asociadas al desarrollo de la obesidad pediátrica.

La adiponectina de la LH está presente en concentraciones más altas en relación con otras adipocinas de la leche (leptina, grelina) y ha sido relacionada tanto positiva como negativamente con el riesgo de obesidad y adiposidad en la vida posterior en lactantes amamantados. La leptina es un factor importante que regula el control del peso corporal infantil. En ratones adultos, la leptina gástrica y el receptor de leptina intestinal aumentan la expresión de ARN mensajero (ARNm) de péptidos antimicrobianos intestinales, independientes de la ingesta de alimentos.

Además, la obesidad materna puede afectar de manera negativa el microbioma intestinal tempranamente; sin embargo, la insulina de la LH y la leptina se asocian con un metabolismo microbiano beneficioso. En resumen, la lactancia materna promueve un normal desarrollo de la microbiota, que mejora la salud de los niños y niñas actual y futura, y tiene también un impacto demostrado en la salud en la vida adulta. ${ }^{36}$

\section{Developmental Origins of Health and Disease y actividad física}

Tanto la actividad como la inactividad física de la madre o de la progenie en etapas tempranas formarían parte de los factores que podrían generar modificaciones en el epigenoma. La inactividad física ha sido asociada a las principales ENT de la vida moderna. ${ }^{37}$ Ante la epidemia de obesidad y DM2, la inactividad física aumenta el riesgo de enfermedad coronaria, accidente vascular cerebral, hipertensión arterial y osteoporosis.
Como punto de partida, es necesario señalar que la inactividad física es fisiológicamente anormal; por lo tanto, su contraparte, la actividad, es una condición sine qua non para mantener un estado de vida saludable.

Según Booth, el estilo de vida sedentario prevalente hoy en día contradice directamente una de las fuerzas naturales que condiciona la evolución de nuestros genes. ${ }^{38}$ Sobre este concepto, se basa la teoría, postulada por algunos autores, que sostiene que la selección de la mayoría de nuestro genoma se llevó a cabo durante la era paleolítica, en la cual los humanos sobrevivían como cazadores-recolectores. Su vida era físicamente activa y sometida a un constante estrés. A su vez, alternaban períodos en los que podían alimentarse con otros en los que pasaban hambre.

Surge la definición del "genoma ahorrador" (thrifty genotype, en inglés), que haría más eficiente la ingesta, la utilización y la reserva de los alimentos. Barker y cols., postulan la teoría del "fenotipo ahorrador", basándose en la hipótesis de que el feto, ante un medio intrauterino adverso, se adaptaría y maximizaría la utilización del escaso aporte de nutrientes. ${ }^{39}$

Booth, extiende la teoría y postula que la supervivencia dependería no solamente de la relación entre la saciedad y el hambre, sino también de los ciclos de actividad e inactividad física; por lo tanto, la selección de un genotipo ahorrador también es sustentada por la actividad física. ${ }^{39,40}$ En definitiva, la influencia ambiental (actividad/inactividad física) que seleccionó determinados genes durante la evolución (genotipo ahorrador), unida a los cambios epigenéticos que ocurren durante la vida temprana (fenotipo ahorrador), condicionarían el estado de salud en su vida adulta.

\section{Epigenómica del ejercicio}

La actividad física ha demostrado un impacto positivo en el tratamiento de las ENT; sin embargo, existe una variación en las respuestas fisiológicas entre las personas frente a un mismo plan de ejercicio. Las diferencias interindividuales podrían atribuirse, en parte, a factores genéticos.

Algunos trabajos ${ }^{41,42}$ han estudiado la influencia del genotipo en la respuesta al ejercicio en paciente con DM2. Sería necesario ampliar las investigaciones, en este sentido, para poder realizar algunas conclusiones específicas. Las variaciones ambientales inducidas por nutrientes han demostrado que generan cambios 
epigenéticos en las etapas críticas del desarrollo. Los estudios relacionados con los efectos del ejercicio son escasos, $y$, además, hay que considerar la dificultad de su interpretación, ya que muy pocos logran aislar el efecto del ejercicio de otras variables, como la dieta. Las modificaciones epigenéticas postuladas por la inducción del "eustress" o "estrés bueno" del ejercicio generarían diferentes tipos de micro-ARN asociados al impacto positivo sobre diferentes patologías, dentro de las cuales se incluye el cáncer. ${ }^{42}$

Estudios en ratas han demostrado que existe evidencia de que el ejercicio físico de la madre puede inducir la sobreexpresión de genes en el hipocampo de ratas, lo cual se traduce en un aumento del aprendizaje espacial. ${ }^{43}$ Lee y cols., basados en la natación de la madre rata durante el embarazo, han mostrado incrementar significativamente la expresión de ARNm del factor neurotrófico derivado del cerebro (brainderived neurotrophic factor; BDNF, por sus siglas en inglés), potenciar la neurogénesis en el hipocampo y mejorar la capacidad de memoria a corto plazo en su progenie. ${ }^{44}$

McCullough y cols., publicaron el primer estudio de una cohorte de 484 pares de madrehijo que relacionó la actividad física prenatal, el peso de nacimiento y los niveles de metilación del ADN en 4 regiones reguladoras del genoma en los RN que influían en el crecimiento y el desarrollo fetal. Este estudio pudo relacionar positivamente la actividad física materna prenatal con el peso al nacer. Si bien no se saben los efectos de estos cambios a largo plazo sobre enfermedades crónicas, podría ser el inicio para interpretar el rol de la actividad física prenatal en las modificaciones de la salud posnatal. ${ }^{45}$

Durante el período posnatal, el músculo esquelético está en un proceso de desarrollo activo, debido a la evolución de la conducta motora, la cual podría modelar la respuesta adulta del músculo esquelético, en la medida en que, en este órgano, existan células mitóticamente activas que puedan heredar los patrones epigenéticos adquiridos durante esta etapa, de manera tal que sean estos mecanismos los responsables de generar mayor riesgo de DM2 en los sujetos sedentarios.

La falta de actividad física en estos períodos podría determinar un patrón de expresión génica que, durante la vida adulta, generaría una respuesta alterada frente a un ambiente que incluyera estilos nocivos de vida. ${ }^{46}$ La presencia de un "fenotipo quieto", caracterizado por la falta de estímulos desencadenados por el ejercicio o actividad motora durante etapas tempranas de desarrollo, condicionaría a un adulto sedentario, el cual, frente al exceso de ingesta con alto contenido calórico, expresaría un fenotipo adulto metabólicamente desregulado y con mayor susceptibilidad de padecer ENT. ${ }^{47}$

\section{Developmental Origins of Health and} Disease y experiencias adversas en la infancia

Las experiencias infantiles adversas (adverse childhood experiences; $A C E$, por sus siglas en inglés) son potencialmente traumáticas y pueden causar un impacto negativo en la salud física y mental. Se ha demostrado que el estrés tóxico y prolongado en la infancia tiene consecuencias para toda la vida en la salud y el bienestar de una persona. Puede interrumpir el desarrollo temprano del cerebro y comprometer el funcionamiento de los sistemas nervioso e inmune. ${ }^{48}$

Se describen como ACE el abuso y maltrato (ya sea físico, sexual, emocional/verbal), la negligencia (física y emocional/psicológica) y situaciones de disfunción en el hogar, como enfermedad mental de los padres, divorcio o separación, violencia doméstica, abuso de alcohol o drogas y encarcelamiento de un miembro de la familia. Existen otras circunstancias estresantes que también deben considerarse posibles $A C E$, debido a la evidencia de sus efectos sobre el desarrollo y la salud del niño. Algunas se identifican con dificultades económicas graves, hambre, discapacidad, guerra, desastres, falta de vivienda, victimización por bullying y discriminación.

El estrés "tóxico" que provocan estas situaciones se asocia con modificaciones en la expresión génica y trastornos del desarrollo cognitivo y social-emocional, y tienen efectos acumulativos que impactarán negativamente en la salud en el curso de la vida. Son más proclives en la edad adulta a padecer enfermedad mental, abuso de sustancias y suicidio, diabetes, enfermedad cardiovascular y cáncer. ${ }^{49-51}$ Está demostrado que los niños que han experimentado, al menos, una $A C E$ tienen un mayor riesgo de problemas crónicos de salud física durante la infancia y adolescencia (por ejemplo, obesidad, trastornos del aprendizaje y del desarrollo), retrasos y problemas mentales, emocionales y alteraciones en la conducta. ${ }^{52}$

Estudios por imágenes llevados a cabo en Bangladesh dan cuenta también de cómo incide 
la pobreza en la arquitectura y funcionalidad cerebral. Se demostró que los niños que crecían en la pobreza tenían volúmenes más pequeños de materia gris que los bebés sin retraso en el crecimiento, y, a su vez, este hallazgo se asoció con peores puntajes en las pruebas de lenguaje y memoria visual a los seis meses. ${ }^{53}$ Habida cuenta de las observaciones resultantes en el desarrollo infantil con los avances tecnológicos y las neurociencias, debería contemplarse la investigación de $A C E$ en la consulta pediátrica e implementar intervenciones para la reducción del estrés tóxico.

\section{Developmental Origins of Health and Disease y adolescencia}

Uno de los interrogantes de interés para los profesionales que atienden a adolescentes es qué papel tienen la genética y el ambiente en el desarrollo cerebral, desde el inicio de la vida hasta haberse completado la adolescencia. Rothman, aseveró que lo genético y lo ambiental estaban siempre presentes y que todas las características humanas, normales y patológicas, eran producto de la interacción entre el medioambiente y los genes: "Todos los rasgos humanos son el $100 \%$ genéticos y el $100 \%$ ambientales". ${ }^{54}$

El pionero de la psiquiatría social, León Eisenberg, señaló lo siguiente: “La expresión génica está ligada al contexto medioambiental: los genes marcan los límites de lo posible, el medioambiente determina lo que se manifiesta en la realidad. La epidemiología de las enfermedades en las poblaciones humanas continuará reflejando dónde y cómo vive la gente, el aire que respiran, el agua que beben, lo que comen, la energía que gastan, las ocupaciones que tienen, el status que tienen en el orden social de sus comunidades, si están socialmente aisladas o rodeados de amigos y familia, y la calidad y cantidad de atención médica que reciben. El desarrollo en genómica servirá para subrayar la importancia de lo social en la fisiopatología de las enfermedades" .55,56

Víctor Penchaszadeh, refiere que es difícil definir lo que es "normal" y "patológico", dados los prejuicios sociales y culturales sobre diversidad humana, y desarrollar investigaciones capaces de aprehender la complejidad humana sin recurrir a reduccionismos de ninguna naturaleza. ${ }^{57}$ En particular, es necesario tener en cuenta que el reduccionismo genético ha causado y continúa causando mucho daño, tanto al conocimiento como a las personas que sufren sus consecuencias de discriminación y estigmatización por poseer rasgos de cualquier tipo ("genético" o "ambiental") que la hacen diferente.

No cabe duda de que, actualmente, el péndulo del interés científico está inclinado a desentrañar los fenómenos de natura por sobre los de nurtura, sesgo que se explica por razones económicas, políticas y de relaciones de poder social, y no por razones científicas. Sin embargo, es totalmente imposible analizar la condición humana dicotomizando lo genético de lo socialambiental..$^{58,41}$

La adolescencia se caracteriza por rápidos cambios biológicos, emocionales y sociales, en la que se desarrollan las capacidades necesarias para una vida productiva, saludable y plena. El alcance del desarrollo potencial del adolescente no depende solo de los genes. Hay factores ambientales que pueden incidir positiva o negativamente. Si el niño vivió en un ambiente con múltiples carencias, que no pudo sostenerlo ni acompañarlo, ya sea por fallas tempranas en los vínculos familiares y/o por sucesivas frustraciones escolares y sociales, pueden aparecer diferentes expresiones de desamparo, como, por ejemplo, "conductas de autodestrucción o antisociales". ${ }^{59}$

El ingreso paulatino del adolescente al mundo adulto se va facilitando cuanto más amplios son los intereses, cuantas más oportunidades ha tenido en el desarrollo de sus capacidades emocionales, cognitivas, sociales y espirituales, y si existen, en su contexto, posibilidades concretas de inserción social, a través del estudio o del trabajo. Y, en todo este potencial desarrollo, intervienen la genética y el ambiente, $\mathrm{y}$, entre ellos, se interpone la epigenética.

\section{Cambios cerebrales en la adolescencia}

Desde los 11 años, el adolescente va adquiriendo la aptitud de pensar hipotéticamente y generalizar a través de observaciones empíricas y de formular conceptos abstractos que sirven para orientar su futura capacidad de decisión. Progresa su capacidad cognitiva hacia una mayor capacidad de abstracción y flexibilidad para la resolución de problemas que caracterizan las operaciones formales. ${ }^{60}$

Durante el proceso adolescente, el desarrollo moral avanza de la etapa preconvencional, típica de los niños menores de 9 años, enfocada en su propio interés, cuando obedecen las normas para evitar el castigo, hacia la convencional. Aquí, la persona tiene en cuenta la perspectiva del otro y 
necesita de la aprobación del afuera. Pocos son los que llegan al nivel posconvencional, más basado en principios humanitarios que en la necesidad de agradar. ${ }^{61}$

Durante mucho tiempo, se creyó que el desarrollo cerebral tenía lugar, fundamentalmente, en los primeros años de vida. Hoy se ha observado, a través de estudios por imágenes, que el desarrollo cerebral continúa aun, pasando los 20 años y este depende de tres procesos: 1) La proliferación que comprende el rápido crecimiento neuronal-glial y la formación de nuevas conexiones sinápticas. 2) La eliminación selectiva o poda de las sinapsis menos eficientes. 3) La mielinización que envuelve los axones para facilitar y hacer más rápida y estable la transmisión neuronal entre diferentes partes del SNC. ${ }^{62}$

Después del pico de crecimiento de la sustancia gris en la adolescencia, se observa una declinación y se supone que aquellas conexiones que no se usan son eliminadas a la manera de una poda. La hipótesis es que el gran potencial de desarrollo, tanto cognitivo, del razonamiento, de las interacciones interpersonales, del control de las emociones, de la motivación, así como la capacidad de anticipar el riesgo en la adolescencia, dependerá de cuáles serán las sinapsis o las conexiones que se eliminarán, lo cual pareciera estar en relación con usarlas o perderlas, y de las que perdurarán. Prevalecerán aquellas que sean utilizadas.

Se ha descubierto cómo la región más evolucionada del cerebro, la corteza prefrontal, encargada de las funciones intelectuales, la conducta dirigida a objetivos y la adquisición de nuevos conocimientos, sufre modificaciones en el ADN de forma muy selectiva y dinámica desde el desarrollo fetal hasta el final de la adolescencia, período en el que las conexiones o sinapsis entre las células nerviosas están aumentando rápidamente. Las modificaciones "epigenéticas" determinan cómo se manifestará el ADN, por medio de marcas químicas que señalan qué genes se activarán y cuáles no. ${ }^{63}$

Además de la metilación del ADN presente en todas las células del organismo desde el nacimiento, incluidas las neuronas y las células de glía, se observó que había una segunda forma de metilación del ADN, denominada "no-CG", que era casi exclusiva de las neuronas y se incrementaba a medida que el cerebro maduraba, con un ritmo máximo hasta los dos años y otro pico en la adolescencia, por lo que se convertía en la forma dominante de metilación del genoma de las neuronas. Estos hallazgos demuestran que el período durante el cual los circuitos neuronales del cerebro maduran va acompañado de un proceso paralelo de reconfiguración a gran escala del epigenoma neuronal. ${ }^{64}$

Cada vez más estudios relacionan las experiencias de los primeros años de vida con las patologías y carencias desarrolladas durante la vida adulta, una cuestión que, tal vez, se relacione con este mecanismo ahora descubierto. ¿Cuánto de lo que somos es, en parte, lo que fueron nuestros padres o es adquirido, no genético? Los patrones de actividad cerebral son muy plásticos y esa plasticidad se relaciona con la metilación del ADN. Y, si estas modificaciones químicas se pueden cambiar de forma exógena, a través de la alimentación, como han demostrado algunos estudios con gemelos, quizá las experiencias tempranas también cambien la actividad cerebral de los niños a través de este mecanismo ahora descubierto. ${ }^{63}$

\section{CONCLUSIONES}

Los recientes avances en la denominada ciencia del desarrollo nos ofrecen una oportunidad sin precedentes para accionar en los mil primeros días de vida y prevenir algunos de los problemas más acuciantes de la salud pública, como son las ENT y los trastornos del desarrollo. La comprensión de la influencia de las experiencias tempranas de los niños, incluso las acontecidas desde antes de la concepción, sobre los resultados en la salud para toda su vida y aún en la de las futuras generaciones, proporciona una base sólida sobre la cual debería enfocarse la inversión económica de los países, la planificación de políticas públicas y la atención en salud, dando prioridad a la embarazada, la infancia y la adolescencia.

Un enfoque en las adecuadas necesidades nutricionales, un entorno de crianza favorable, la prevención de las exposiciones a tóxicos ambientales y a estrés crónico desde los comienzos de la vida deberían ser los pilares para mejorar la calidad de vida de los niños y las familias, prevenir las ENT y favorecer el desarrollo pleno. La complejidad de los mecanismos fisiopatogénicos de diversos estímulos ambientales que modifican la programación fetal y aumentan la susceptibilidad a enfermedades en la vida adulta, develada a nivel biomolecular con gran celeridad, no son objeto de esta actualización. 


\section{REFERENCIAS}

1. Organización Panamericana de la Salud. Las ENT de un vistazo: Mortalidad de las enfermedades no transmisibles y prevalencia de sus factores de riesgo en la Región de las Américas. Washington, DC: OPS; 2019.

2. Heindel J, Balbus J, Birnbaum L, Brune-Drisse MN, et al. Developmental Origins of Health and Disease: Integrating Environmental Influences. Endocrinology. 2015; 156(10):3416-21.

3. National Research Council, Institute of Medicine, Committee on Integrating the Science of Early Childhood Development, Shonkoff JP, Phillips D (eds.). From Neurons to Neighborhoods: The Science of Early Childhood Development. Washington, DC: National Academies Press; 2000.

4. Haugen A, Schug T, Collman G, Heindel J. Evolution of DOHaD: The impact of environmental health sciences. J Dev Orig Health Dis. 2015; 6(2):55-64.

5. LillycropK, Burdge G. Epigenetic mechanisms linking early nutrition to long term health. Best Pract Res Clin Endocrinol Metab. 2012; 26(5):667-76.

6. Williams T, Drake A. What a general paediatrician needs to know about early life programming. Arch Dis Child. 2015; 100(11):1058-63.

7. Wang X. Early life programming and metabolic syndrome. World J Pediatr. 2013; 9(1):5-8.

8. Forsdahl A. Are poor living conditions in childhood and adolescence an important risk factor for arteriosclerotic heart disease? Br J Prev Soc Med. 1977; 31(2):91-5.

9. Roseboom T, Van Der Meulen J, Ravelli A, Osmond C, et al. Perceived health of adults after prenatal exposure to the Dutch famine. Paediatr Perinat Epidemiol. 2003; 17(4):391-7.

10. Yajnik C, Deshmukh U. Fetal programming: maternal nutrition and role of one-carbon metabolism. Rev Endocr Metab Disord. 2012; 13(2):121-7.

11. Yajnik C, Fall C, Vaidya U, Pandit A, et al. Fetal growth and glucose and insulin metabolism in four-year-old Indian children. Diabet Med. 1995; 12(4):330-6.

12. Bavdekar A, Yajnik C, FallC, BapatS, et al. Insulin resistance syndrome in 8-year-old Indian children: small at birth, big at 8 years, or both? Diabetes. 1999; 48(12):2422-9.

13. Rao S, Yajnik CS, Kanade A, Fall CH, et al. Intake of micronutrient-rich foods in rural Indian mothers is associated with the size of their babies at birth: Pune Maternal Nutrition Study. J Nutr. 2001; 131(4):1217-24.

14. Bhate V, Deshpande S, Bhat D, Joshi N, et al. Vitamin B12 status of pregnant Indian women and cognitive function in their 9-year-old children. Food Nutr Bull. 2008; 29(4):249-54.

15. Smith J, Cianflone K, Biron S, Hould FS, et al. Effects of maternal surgical weight loss in mothers on intergenerational transmission of obesity. J Clin Endocrinol Metab. 2009; 94(11):4275-83.

16. Hales CN, Barker DJ, Clark PM, Cox LJ, et al. Fetal and infant growth and impaired glucose tolerance at age 64 . BMJ. 1991; 303(6809):1019-22.

17. Crowther NJ, Cameron N, Trusler J, Gray IP. Association between poor glucose tolerance and rapid post natal weight gain in seven-year-old children. Diabetologia. 1998; 41(10):1163-7.

18. Eriksson JG, Osmond C, KajantieE, Forsen TJ, et al. Patterns of grow th among children who later develop type 2 diabetes or its risk factors. Diabetologia. 2006; 49(12):2853-8.

19. Koletzko B, Von Kries R, Closa R, Escribano J, et al. Lower protein in infant formula is associated with lower weight up to age 2 y: a randomized clinical trial. Am J Clin Nutr. 2009; 89(6):1836-45.

20. Singhal A, Kennedy K, Lanigan J, Fewtrell M, et al. Nutrition in infancy and long-term risk of obesity: evidence from 2 randomized controlled trials. Am J Clin Nutr. 2010;
92(5):1133-44.

21. Mericq V, Martinez-Aguayo A, Uauy R, Iñiguez G, et al. Long-term metabolic risk among children born premature or small for gestational age. Nat Rev Endocrinol. 2017; 13(1):50-62.

22. ACOG Committee Opinion No. 575. Exposure to toxic environmental agents. Obstet Gynecol. 2013; 122(4):931-5.

23. Stiemsma LT, Michels KB. The Role of the Microbiome in the Developmental Origins of Health and Disease.Pediatrics. 2018; 141(4):e20172437.

24. Van de Wiele T, Vanhaecke L, Boeckaert C, Peru K, et al. Human colon microbiota transform polycyclic aromatic hydrocarbons to estrogenic metabolites. Environ Health Perspect. 2005; 113(1):6-10.

25. Van den Brule S, Ambroise J, Lecloux H, Levard C, et al. Dietary silver nanoparticles can disturb the gut microbiota in mice. Part Fibre Toxicol. 2016; 13(1):38.

26. Gao B, Chi L, Mahbub R, Bian X, et al. Multi-omics reveals that lead exposure disturbs gut microbiome development, key metabolites, and metabolic pathways. Chem Res Toxicol. 2017; 30(4):996-1005.

27. Lu K, Abo RP, Schlieper KA, Graffam ME, et al. Arsenic exposure perturbs the gut microbiome and its metabolic profile in mice: an integrated metagenomics and metabolomics analysis. Environ Health Perspect. 2014; 122(3):284-91.

28. Koestel ZL, Backus RC, Tsuruta K, Spollen WG, et al. Bisphenol A (BPA) in the serum of pet dogs following short-term consumption of canned dog food and potential health consequences of exposure to BPA. Sci Total Environ. 2017; 579:1804-14.

29. García-Arévalo M, Alonso-Magdalena P, Servitja JM, Boronat-Belda T, et al. Maternal Exposure to Bisphenol-A during pregnancy increases pancreatic beta-cell growth during early life in male mice offspring. Biomed Res Int. 2016; 157(11):4158-71.

30. Borre YE, O'keeffe GW, Clarke G, Stanton C, etal. Microbiota and Neurodevelopmental windows: implications for brain disorders. Trends Mol Med. 2014; 20(9):509-18.

31. Alonso C, Vicario M, Pigrau M, Lobo B, et al. Intestinal barrier function and the brain-gut axis. Adv Exp Med Biol. 2014; 817:73-113.

32. Ashida H, Ogawa M, Kim M, Mimuro H, et al. Bacteria and host interactions in the gut epithelial barrier. Nat Chem Biol. 2011; 8(1):36-45.

33. Le Doare K, Holder B, Bassett A, Pannaraj PS. Mother's Milk: a purposeful contribution to the development of the infant microbiota and immunity. Front Immunol. 2018; 9:361.

34. Korpela K, Salonen A, Virta L, Kekkonen RA, et al. Association of early-life antibiotic use and protective effects of breastfeeding role of the intestinal microbiota. JAMA Pediatr. 2016; 170(8):750-7.

35. Lemas D, Yee S, Cacho N, Miller D, et al. Exploring the contribution of maternal antibiotics and breastfeeding to development of the infant microbiome and pediatric obesity. Semin Fetal Neonatal Med. 2016; 21(6):406-9.

36. Victora C, Bahl R, Barros A, França G, et al. Breastfeeding in the 21st century: epidemiology, mechanisms, and lifelong effect. Lancet. 2016; 387(10017):475-90.

37. Booth FW, Lees SJ. Fundamental questions about genes, inactivity, and chronic diseases. Physiol Genomics. 2007; 28(2):146-57.

38. Booth F, Gordon S, Carlson C, Hamilton M. Waging war on modern chronic diseases: primary prevention through exercise biology. J Appl Physiol (1985). 2000; 88(2):774-87.

39. Yajnik C. Commentary: Thrifty phenotype: 20 years later. Int J Epidemiol. 2013; 42(5):1227-9. 
40. McKenzie JA, Weiss EP, Ghiu IA, Kulaputana O, et al. Influence of the interleukin-6-174G/C gene polymorphism on exercise training-induced changes in glucose tolerance indexes. J Appl Physiol (1985). 2004; 97(4):1338-42.

41. Adamo KB, Sigal RJ, Williams K, Kenny G, et al. Influence of Pro12Ala peroxisome proliferator-activated receptor gamma2 polymorphism on glucose response to exercise training in type 2 diabetes. Diabetologia. 2005; 48(8):1503-9.

42. Ntanasis-Stathopoulos J, Tzanninis A, Philippou M, Koutsilieris P. Epigenetic regulation on gene expression induced by physical exercise. J Musculoskelet Neuronal Interact. 2013; 13(2):133-46.

43. ParnpiansilP, JutapakdeegulN, ChentanezT, Kotchabhakdi N.Exercise during pregnancy increases hippocampal brainderived neurotrophic factor mRNA expression and spatial learning in neonatal rat pup. Neurosci Lett. 2003; 352(1):45-8.

44. Lee HH, Kim H, Lee JW, Kim YS, et al. Maternal swimming during pregnancy enhances short-term memory and neurogenesis in the hippocampus of rat pups. Brain Dev. 2006; 28(3):147-54.

45. McCullough L, Mendez M, Miller E, Murtha A, et al. Associations between prenatal physical activity, birth weight, and DNA methylation at genomically imprinted domains in a multiethnic newborn cohort. Epigenetics. 2015; 10(7):597-606.

46. Alegría-Torres J, Baccarelli A, Bollati V. Epigenetics and lifestyle. Epigenomics. 2011; 3(3):267-77.

47. Zhang F, Cardarelli R, Carroll J, Zhang S, et al. Physical activity and global genomic DNA methylation in a cancerfree population. Epigenetics. 2011; 6(3):293-9.

48. Felitti VJ, Anda RF, Nordenberg D, Willamson DF, et al. Relationship of childhood abuse and household dysfunction to many of the leading causes of death in adults: The Adverse Childhood Experiences (ACE) Study. Am J Prev Med. 1998; 14(4):245-58.

49. PechtelP,PizzagalliDA.Effects of early lifestress on cognitive and affective function: an integrated review of human literature. Psychopharmacology (Berl). 2011; 214(1):55-70.

50. Ports KA, Merrick MT, Stone DM, Wilkins NJ, et al. Adverse childhood experiences and suicide risk: toward comprehensive prevention. Am J Prev Med. 2017; 53(3):400-3.

51. Sonu S, Post S, Feinglass J. Adverse childhood experiences and the onset of chronic disease in young adulthood. Prev Med. 2019; 123:163-70.
52. Garner AS, ShonkoffJP, Committe on Psychosocisl Aspects of Child and Family Health, Committe on Early Childhood, Adoption, and Dependent Care, Section on Developmental and Behavioral Pediatrics. Early childhood adversity, toxic stress, and the role of the pediatrician: translating developmental science into lifelong health. Pediatrics. 2012; 129(1):e224-31.

53. Pavlakis A, Noble K, Pavlakis S, Ali N, et al. Brain imaging and electrophysiology biomarkers: Is there a role in poverty and education outcome research? Pediatr Neurol. 2015; 52(4):383-8.

54. Rothman KJ, Greenland S. Causation and causal inference in epidemiology. Am J Public Health. 2005; 95(Suppl 1):S144-50.

55. Link BG, Phelan J. Social conditions as fundamental causes of disease. J Health Soc Behav. 1995; Spec No:80-94.

56. Eisenberg N, Zhou Q, Spinrad TL, ValienteC, etal. Relations among positive parenting, children's effortful control, and externalizing problems: a three $\square$ wave longitudinal study. Child Dev. 2005; 76(5):1055-71.

57. Penchaszadeh VB. Reflexiones de un genetista sobre la influencia de los genes en los trastornos psiquiátricos. $\operatorname{VERTEX~(B.~Aires).~2016;~27(129):357-67.~}$

58. Holtz TH, Holmes SM, Stonington S, Eisenberg L. Health is still social: contemporary examples in the age of the genome. PLoS Med. 2006; 3(10):e419.

59. Winnicott DW. Communicating and not communicating leading to a study of certain opposites. In: Winnicott DW. The maturational process and the facilitating environment. London: Karnac; 1990.Págs.179-92.

60. Pasqualini D, Llorens A. Manual de adolescencia y salud. Un abordaje integral. Buenos Aires: Journal; 2016.

61. Kohlberg L. Essays in moral development. Vol. 2. The psychology of moral development: Moral stages and the life cycle. San Francisco: Harper and Row. 1976.

62. Giedd JN, Blumenthal J, Jeffries NO, Castellanos FX, et al. Brain development during childhood and adolescence: a longitudinal MRI study. Nat Neurosci. 1999; 2(10):861-3.

63. Bernardo A. ¿Qué necesita alguien para hacerse mayor? Hipertextual. 2013. [Acceso: 19 de febrero de 2020]. Disponibleen:https: / / hipertextual.com/2013/07/ recetahacerse-mayor.

64. Lister R, Mukamel EA, Nery JR, Urich M, et al. Global epigenomic reconfiguration during mammalian brain development. Science. 2013; 341(6146):1237905. 